\title{
Upregulation of Phosphatase 1 Nuclear-Targeting Subunit (PNUTS) Is an Independent Predictor of Poor Prognosis in Prostate Cancer
}

\author{
Andreas Marx, ${ }^{1}$ Andreas M. Luebke, ${ }^{2}$ Till S. Clauditz, ${ }^{2}$ Stefan Steurer, ${ }^{2}$ Christoph Fraune, ${ }^{2}$ \\ Claudia Hube-Magg, ${ }^{2}$ Franziska Büscheck, ${ }^{2}$ Doris Höflmayer, ${ }^{2}$ Maria Christina Tsourlakis, ${ }^{2}$ \\ Christina Möller-Koop, ${ }^{2}$ Ronald Simon ${ }^{1},{ }^{2}$ Guido Sauter, ${ }^{2}$ Cosima Göbel, ${ }^{2}$ Patrick Lebok, ${ }^{2}$ \\ David Dum, ${ }^{2}$ Simon Kind, ${ }^{2}$ Sarah Minner, ${ }^{2}$ Jakob Izbicki, ${ }^{3}$ Thorsten Schlomm, \\ Hartwig Huland, ${ }^{5}$ Hans Heinzer, ${ }^{5}$ Eike Burandt, ${ }^{2}$ Alexander Haese, ${ }^{5}$ Markus Graefen, ${ }^{5}$ \\ and Jan Meiners ${ }^{3}$ \\ ${ }^{1}$ Institute of Pathology, Clinical Center Fürth, Jakob-Henle-Straße 1, 90766 Fürth, Germany \\ ${ }^{2}$ Institute of Pathology, University Medical Center Hamburg-Eppendorf, 20246 Hamburg, Germany \\ ${ }^{3}$ General, Visceral and Thoracic Surgery Department and Clinic, University Medical Center Hamburg-Eppendorf, \\ 20246 Hamburg, Germany \\ ${ }^{4}$ Department of Urology, Charité-Universitätsmedizin Berlin, 10117 Berlin, Germany \\ ${ }^{5}$ Martini-Clinic, Prostate Cancer Center, University Medical Center Hamburg-Eppendorf, 20246 Hamburg, Germany \\ Correspondence should be addressed to Ronald Simon; r.simon@uke.de
}

Received 13 September 2019; Revised 23 January 2020; Accepted 7 April 2020; Published 25 April 2020

Academic Editor: Leigh A. Madden

Copyright (C) 2020 Andreas Marx et al. This is an open access article distributed under the Creative Commons Attribution License, which permits unrestricted use, distribution, and reproduction in any medium, provided the original work is properly cited.

Protein phosphatase 1 nuclear-targeting subunit (PNUTS) is ubiquitously expressed and associates with PTEN and protein phosphatase 1 (PP1) to control its activity. The role of PNUTS overexpression has hardly been studied in cancer. In this study, we used immunohistochemistry to quantitate PNUTS expression on a tissue microarray containing 17,747 clinical prostate cancer specimens. As compared to normal prostate epithelium, PNUTS expression was often higher in cancer. Among 12,235 interpretable tumors, PNUTS staining was negative in $21 \%$, weak in $34 \%$, moderate in $35 \%$, and strong in $10 \%$ of cases. High PNUTS expression was associated with higher tumor stage, classical and quantitative Gleason grade, nodal stage, surgical margin, Ki67 labeling index, and early biochemical recurrence $(p<0.0001$ each). PNUTS expression proved to be a moderate prognostic parameter with a maximal univariable Cox proportional hazard for PSA recurrence-free survival of 2.21 compared with 5.91 for Gleason grading. It was independent from established prognostic parameters in multivariable analysis. Comparison with molecular data available from earlier studies using the same TMA identified associations between high PNUTS expression and elevated androgen receptor expression $(p<0.0001)$, presence of TMPRSS2:ERG fusion $(p<0.0001)$, and 8 of 11 chromosomal deletions (3p13,5q21, 8p21, 10q23, 12p13, 13q14, 16q24, and 17p13; $p<0.05$ each). Particularly strong associations with PTEN and 12p13 deletions $(p<0.0001$ each) may indicate a functional relationship, which has already been established for PNUTS and PTEN. PNUTS had no additional role on outcome in PTEN-deleted cancers. In conclusion, the results of our study identify high PNUTS protein levels as a predictor of poor prognosis possibly linked to increased levels of genomic instability. PNUTS measurement, either alone or in combination, might be of clinical utility in prostate cancers. 


\section{Introduction}

In a recent overview from Bray et al., prostate cancer is the most common cancer in males in the majority of countries in the world [1]. The clinical course is highly variable, and active surveillance and watchful waiting are newer concepts in the therapy of prostate cancer [2]. Therefore, we need reliable criteria for the distinction between high-risk and lowrisk patients. It is hoped that molecular prognostic markers will improve this selection.

Protein phosphatase 1 nuclear-targeting subunit (PNUTS) is a bifunctional gene encoding both the PNUTS protein and, by alternative splicing, a long noncoding RNA termed lncRNA-PNUTS [3]. The PNUTS protein is ubiquitously expressed and associates with protein phosphatase 1 (PP1) to control its activity [4]. PP1 is one of the major human protein phosphatases that dephosphorylate hundreds of kinases involved in a wide range of cellular actions [5]. Accordingly, PNUTS is implicated in all PP1-governed processes including RNA processing [6], DNA repair [7], chromatin decondensation [8], and maintenance of telomere stability [9]. PP1 is also involved in growth control through interaction with PTEN, RB1, MYC, and Aurora kinases [1014]. The noncoding transcript lncRNA-PNUTS appears to function as a regulator of epithelial-mesenchymal transition, an essential process for tissue and organ formation, and also for tumor development and metastasis [15]. Several studies suggest that targeting PNUTS may have antitumor activity. For example, PNUTS knockdown increased apoptosis in breast, ovarian, and colon cancers cells [11] and potentiated the apoptotic effect of the cyclin-dependent kinase inhibitor roscovitine in breast and colon cancer cells [16]. Injection of the microRNA miR-34a, a natural downregulator of PNUTS, into glioblastoma xenograft tumors reduced telomere length [17] and targeting PNUTS with miR-383 induced cell cycle arrest in testicular embryonal carcinoma cells [18]. Most recently, it was shown that PNUTS is an essential partner of poly (ADP-ribose) polymerase 1 in DNA repair [19], making PNUTS a potential drug target in the therapy of DNA double-strand repairdeficient tumors.

One report using mRNA expression data from the Oncomine database [20] suggests that PNUTS may be upregulated in prostate cancers as compared to normal prostate tissues [10]. This observation prompted us to further investigate the role of this potentially important gene by analyzing a prostate cancer tissue microarray containing tumor samples from more than 17,000 individual patients.

\section{Materials and Methods}

2.1. Patients. The 17,747 patients had prostatectomy between 1992 and 2015 (Department of Urology and the Martini Clinic at the University Medical Center Hamburg-Eppendorf). Quantitative Gleason grading was performed as described [21]. Follow-up data were available for 14,667 patients with a median follow-up of 48 months. Prostatespecific antigen (PSA) recurrence was defined as the time point when postoperative PSA was at least $0.2 \mathrm{ng} / \mathrm{ml}$ and
TABle 1: Pathological and clinical data of the arrayed prostate cancers.

\begin{tabular}{|c|c|c|}
\hline & \multicolumn{2}{|c|}{ No. of patients (\%) } \\
\hline & $\begin{array}{l}\text { Study cohort } \\
\text { on TMA* }\end{array}$ & $\begin{array}{l}\text { Biochemical } \\
\text { relapse }\end{array}$ \\
\hline Follow-up & 14,464 & $3,612(25 \%)$ \\
\hline Mean/median (month) & $56 / 48$ & - \\
\hline \multicolumn{3}{|l|}{ Age $(y)$} \\
\hline$\leq 50$ & 433 & $66(15 \%)$ \\
\hline $51-59$ & 4,341 & $839(19 \%)$ \\
\hline $60-69$ & 9,977 & $2,073(21 \%)$ \\
\hline$\geq 70$ & 2,936 & $634(22 \%)$ \\
\hline \multicolumn{3}{|l|}{$\begin{array}{l}\text { Pretreatment PSA } \\
(\mathrm{ng} / \mathrm{ml})\end{array}$} \\
\hline$<4$ & 2,225 & $313(14 \%)$ \\
\hline $4-10$ & 10,520 & $1,696(16 \%)$ \\
\hline $10-20$ & 3,662 & $1,043(29 \%)$ \\
\hline$>20$ & 1,231 & $545(44 \%)$ \\
\hline \multicolumn{3}{|l|}{ pT stage (AJCC 2002) } \\
\hline pT2 & 11,518 & $1,212(11 \%)$ \\
\hline pT3a & 3,842 & $1,121(29 \%)$ \\
\hline pT3b & 2,233 & $1,213(54 \%)$ \\
\hline pT4 & 85 & $63(74 \%)$ \\
\hline \multicolumn{3}{|l|}{ Gleason grade } \\
\hline$\leq 3+3$ & 3,570 & $264(7 \%)$ \\
\hline $3+4$ & 9,336 & $1,436(15 \%)$ \\
\hline $3+4$ tertiary 5 & 1,697 & $165(10 \%)$ \\
\hline $4+3$ & 2,903 & $683(24 \%)$ \\
\hline $4+3$ tertiary 5 & 1,187 & $487(41 \%)$ \\
\hline$\geq 4+4$ & 999 & $531(53 \%)$ \\
\hline \multicolumn{3}{|l|}{ pN stage } \\
\hline pNo & 10,636 & $2,243(21 \%)$ \\
\hline $\mathrm{pN}+$ & 1,255 & $700(56 \%)$ \\
\hline \multicolumn{3}{|l|}{ Surgical margin } \\
\hline Negative & 14,297 & $2,307(16 \%)$ \\
\hline Positive & 3,388 & $1,304(39 \%)$ \\
\hline
\end{tabular}

${ }^{*}$ Numbers do not always add up to 17,747 in different categories because of cases with missing data. AJCC: American Joint Committee on Cancer.

increasing at subsequent measurements. Patient characteristics are summarized in Table 1 . The tissue microarray (TMA) manufacturing process was described earlier [22]. A single $0.6 \mathrm{~mm}$ core was taken for each patient. The TMA was annotated with data on Ki67 labeling index (Ki67LI) [23], ERG protein expression and ERG rearrangement analysis by fluorescence in situ hybridization (FISH) [24], and deletion status of 3p13 (FOXP1) [25], 5q21 (CHD1) [26], 6q15 (MAP3K7) [27], 8p21 [28], 10q23 (PTEN) [29], 12p13 [30], $12 \mathrm{q} 24$ [31], 13q14 [32], 16q24 [33], 17p13 [34], and 18q21 [35]. The usage of archived diagnostic leftover tissues for manufacturing of tissue microarrays and their analysis for research purposes as well as patient data analysis has been approved by local laws (HmbKHG, \$12a) and by the local ethics committee (Ethics Commission Hamburg, WF- 


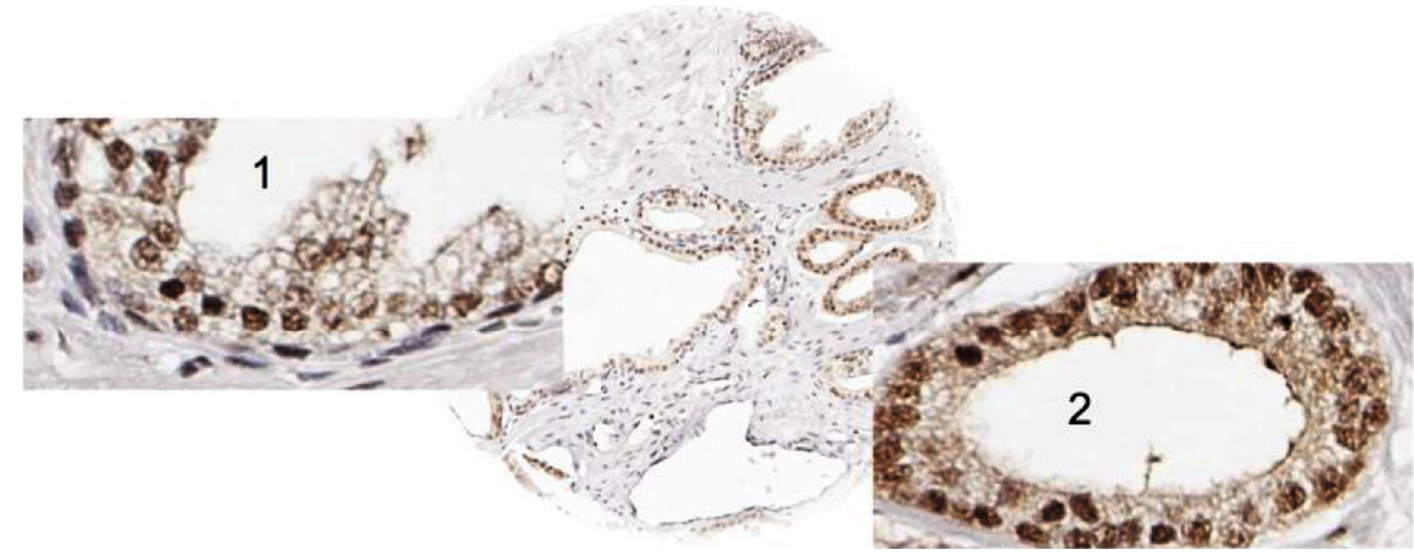

(a)

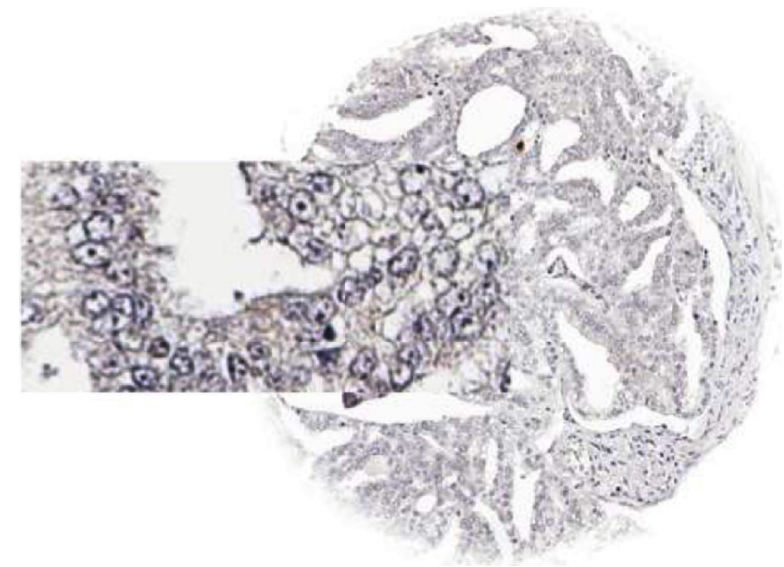

(b)

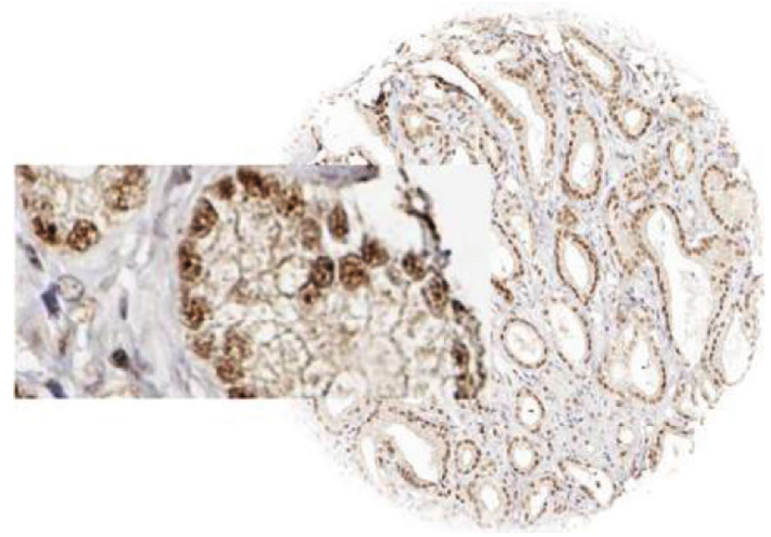

(d)

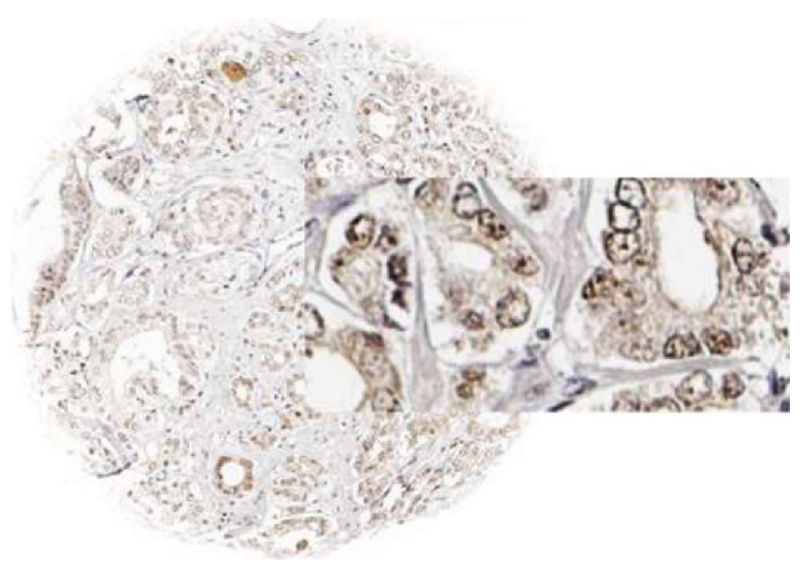

(c)

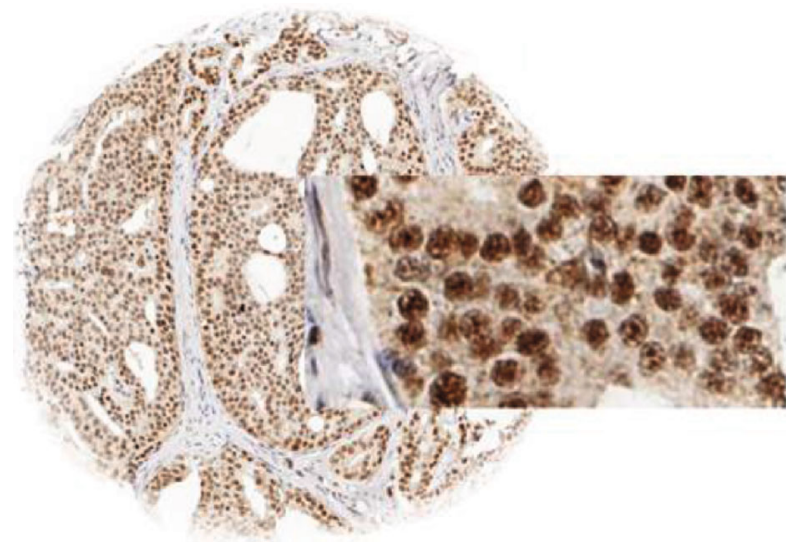

(e)

FIGURE 1: Examples of PNUTS staining: (a) comparison of PNUTS staining in (1) normal and (2) cancerous prostate glands of the same TMA spot. Cancer spots with (b) lack of staining and (c) weak, (d) moderate, and (e) strong staining.

049/09). All work has been carried out in compliance with the Helsinki Declaration.

2.2. Immunohistochemistry. Freshly cut TMA sections were stained in a single experiment, deparaffinized, and exposed to heat-induced antigen retrieval $\left(5 \mathrm{~min}, 121^{\circ} \mathrm{C}, \mathrm{pH} 7.8\right.$ Tris-EDTA buffer). Primary antibody specific for PNUTS (rabbit polyclonal antibody, Novus Biologicals, Centennial,
Colorado, USA, NB100-604; dilution 1:4,050) was applied $\left(37^{\circ} \mathrm{C}, 60 \mathrm{~min}\right.$ ) and visualized with EnVision (Dako, Glostrup, Denmark). Anti-PNUTS showed punctuate staining inside the nuclei that was typically accompanied by weaker cytoplasmic costaining. Because the nuclear staining pattern is compatible with its function and cellular localization [36], only nuclear staining was scored as follows. The staining intensity $(0,1+, 2+$, and $3+)$ as well as the fraction 
TABLE 2: PNUTS expression and cancer phenotype.

\begin{tabular}{|c|c|c|c|c|c|c|}
\hline \multirow{2}{*}{ Parameter } & \multirow{2}{*}{$N$} & \multicolumn{4}{|c|}{ PNUTS expression (\%) } & \multirow[b]{2}{*}{$p$} \\
\hline & & Negative & Weak & Moderate & Strong & \\
\hline All cancers & 12,235 & 21 & 34 & 35 & 10 & \\
\hline Tumor stage & & & & & & $<0.0001$ \\
\hline pT2 & 7,895 & 25 & 36 & 32 & 8 & \\
\hline pT3a & 2,707 & 17 & 30 & 38 & 14 & \\
\hline pT3b-pT4 & 1,590 & 12 & 31 & 42 & 14 & \\
\hline Gleason grade & & & & & & $<0.0001$ \\
\hline$\leq 3+3$ & 2,379 & 34 & 31 & 28 & 7 & \\
\hline $3+4$ & 6,559 & 20 & 36 & 34 & 10 & \\
\hline $3+4$ tertiary 5 & 549 & 15 & 37 & 40 & 8 & \\
\hline $4+3$ & 1,168 & 14 & 31 & 40 & 15 & \\
\hline $4+3$ tertiary 5 & 867 & 10 & 34 & 44 & 13 & \\
\hline$\geq 4+4$ & 619 & 15 & 29 & 42 & 14 & \\
\hline Quantitative Gleason grade & & & & & & $<0.0001$ \\
\hline$\leq 3+3$ & 2,379 & 34 & 31 & 28 & 7 & \\
\hline $3+4 \leq 5 \%$ & 1,682 & 24 & 39 & 30 & 7 & \\
\hline $3+46-10 \%$ & 1,639 & 22 & 36 & 33 & 9 & \\
\hline $3+411-20 \%$ & 1,466 & 18 & 35 & 35 & 12 & \\
\hline $3+421-30 \%$ & 737 & 16 & 33 & 39 & 13 & \\
\hline $3+431-49 \%$ & 570 & 17 & 36 & 37 & 10 & \\
\hline $3+4$ tertiary 5 & 549 & 15 & 37 & 40 & 8 & \\
\hline $4+350-60 \%$ & 501 & 14 & 34 & 39 & 14 & \\
\hline $4+3$ tertiary 5 & 867 & 10 & 34 & 44 & 13 & \\
\hline $4+361-100 \%$ & 521 & 15 & 29 & 40 & 16 & \\
\hline$\geq 4+4$ & 542 & 15 & 31 & 41 & 13 & \\
\hline Lymph node metastasis & & & & & & $<0.0001$ \\
\hline No & 7,347 & 18 & 34 & 37 & 11 & \\
\hline $\mathrm{N}+$ & 856 & 10 & 31 & 44 & 15 & \\
\hline Preoperative PSA level $(\mathrm{ng} / \mathrm{ml})$ & & & & & & 0.0001 \\
\hline$<4$ & 1,469 & 20 & 36 & 33 & 11 & \\
\hline $4-10$ & 7,293 & 21 & 35 & 35 & 9 & \\
\hline $10-20$ & 2,546 & 22 & 33 & 35 & 10 & \\
\hline$>20$ & 854 & 22 & 28 & 37 & 14 & \\
\hline Surgical margin & & & & & & $<0.0001$ \\
\hline Negative & 9,771 & 22 & 35 & 34 & 9 & \\
\hline Positive & 2,423 & 18 & 30 & 39 & 13 & \\
\hline
\end{tabular}

(percentage) of stained cells was recorded for each tissue spot. A final score was built from these two parameters as follows. Lack of any staining (intensity 0) was considered "negative," $1+$ staining in $\leq 70 \%$ of tumor cells or $2+$ staining in $\leq 30 \%$ of tumor cells was considered "weak," $2+$ staining in $>70 \%$ of tumor cells or $2+$ staining in $>30 \%$ but $\leq 70 \%$ of tumor cells or $3+$ staining in $\leq 30 \%$ of tumor cells was considered "moderate," and $2+$ staining in $>70 \%$ of tumor cells or $3+$ staining in $>30 \%$ of tumor cells was considered "strong."

2.3. Statistics. JMP 12 software (SAS Institute Inc., NC, USA) was used for calculations. Contingency tables and the $\chi^{2}$ test were utilized to examine associations between molecular and histopathological tumor parameters. Kaplan-Meier survival curves were analyzed with the log-rank test to detect differences between groups. Cox proportional hazards regression analysis was performed to test for statistical independence between pathological, molecular, and clinical variables.

\section{Results}

3.1. Technical Issues. A total of 12,235 of 17,747 tumor samples $(69 \%)$ were interpretable in our TMA analysis. Reasons for $31 \%$ noninformative cases included lack of tissue samples or absence of unequivocal cancer tissue in the TMA spot. 
3.2. PNUTS Expression in Normal and Cancerous Prostate Tissues. PNUTS staining was predominantly nuclear with a focus on nucleoli resulting in a "punctuated" staining pattern. In 20 cases of normal prostate gland, luminal cells stained weakly positive for PNUTS, while basal cells were negative (Figure $1(\mathrm{a} 1)$ ). In prostate cancers, nuclear staining was seen in 9632 of our 12,235 (78.7\%) interpretable tumors and was considered weak in $33.9 \%$, moderate in $34.7 \%$, and strong in $10.1 \%$ of cases (Figures 1(a2), 1(b), and 1(e)). Increased PNUTS staining showed significant associations with adverse tumor features (Table 2). Strong staining of PNUTS was associated with advanced tumor stage $(p<0.0001)$, high classical and quantitative Gleason grade $(p<0.0001$ each), presence of lymph node metastasis $(p<0.0001)$, high preoperative PSA level $(p=0.0001)$, and positive surgical margin $(p<0.0001)$. PNUTS staining was also linked to early biochemical recurrence (Figure 2). Accordingly, subgroup analyses of cancers with identical Gleason score revealed a prognostic role of PNUTS expression in Gleason $3+4 \quad(p<0.0001)$ and Gleason $4+3$ $(p=0.0002$, Supplementary Figure S2a) as well as in particular quantitative Gleason grades (Supplementary Figure S2d, h).

3.3. PNUTS and TMPRSS2:ERG Fusion Status. Data on both ERG FISH and IHC were available in 4,998 cancers with concordant results in $95 \%$ of cases. PNUTS staining was significantly more prevalent in cancers harboring TMPRSS2:ERG rearrangements than in cancer lacking ERG fusions (Figure 3). Because of these differences, associations of PNUTS with tumor phenotype and PSA recurrence were separately analyzed in ERG-negative and ERG-positive cancers. All these associations with tumor phenotype (Supplementary Tables S1 and S2) and clinical outcome ( $p<0.0001$ each, Supplementary Figure S1) hold true in both subgroups.

3.4. PNUTS and Chromosomal Deletions. For 10 of 11 analyzed deletions, PNUTS staining was stronger in deleted than in nondeleted cancers. This difference reached statistical significance for 8 of the 11 analyzed regions (Supplementary Figure S1). In ERG-negative cancers, a statistically significant difference was also seen for 8 of 11 analyzed deletions (Supplementary Figure S1b). In ERG-positive cancers, a statistically significant difference was found for 5 of 11 deletions (Supplementary Figure S1c). A particularly prominent PNUTS staining was seen in cancers with PTEN (10q23) and $12 \mathrm{p}$ deletions, which was highly significant in all, ERG-negative, and ERG-positive cancers $(p<0.0001$ each).

3.5. PNUTS, Androgen Receptor, and Tumor Cell Proliferation (Ki67LI). In 5,414 cancers with available AR expression data, the intensity of PNUTS staining was highly related to AR levels (Figure 4). This also applied for subsets of ERG-negative and ERG-positive cancers (data not shown). PNUTS staining was significantly linked to increased Ki67LI (Table 3). This held true in most subsets of cancers with identical Gleason score, including Gleason $\leq 3+3$

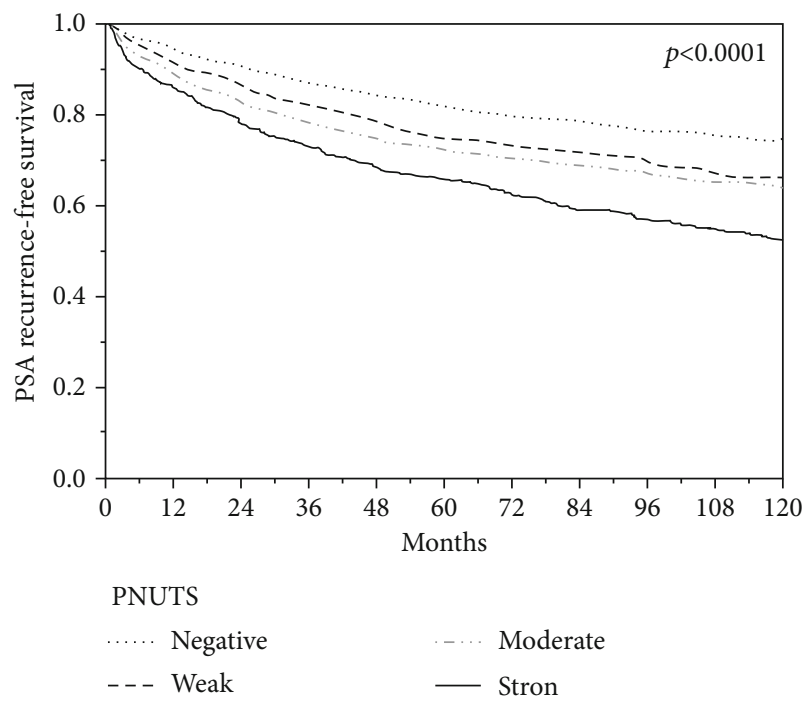

FIgURE 2: Association between PNUTS expression and biochemical recurrence after prostatectomy.

$(p<0.0001), 3+4 \quad(p<0.0001), 3+4$ with tertiary grade $5(p=0.0019)$, and $4+3(p=0.0009)$.

3.6. PSA Recurrence-Free Survival in Uni- and Multivariable Analyses. The univariable analysis showed a moderate Cox proportional hazard ratio of 2.21 for strong versus negative PNUTS expression compared with a hazard ratio of 5.91 for Gleason grading (Table 4). When the five variables of the univariable analysis were combined in the multivariable model, the hazard ratios were reduced but remained significant for each variable indicating that they are in part independent of each other. Strong PNUTS expression was predictive at a comparable level as PTEN deletion. The effect was observed in both ERG subsets (Table 4, Supplementary Table S3).

\section{Discussion}

The results of our study identify high PNUTS protein expression as a predictor of poor prognosis in prostate cancer. The peculiar "punctuated" nuclear staining pattern of PNUTS has already been described in an earlier study [36]. It fits well with the known interaction with PP1, which constitutes an important part of the nucleolar proteome. The PP1 subunit PP1 gamma and to a lesser extent PP1 beta are highly concentrated in the nucleoli of interphase cells [36]. Finding PNUTS expression at higher levels in cancer glands as compared to adjacent normal prostate gland suggests that the protein becomes upregulated during prostate cancer development and progression. Our findings are supported by findings from the Oncomine database, where PNUTS mRNA levels were higher in prostate cancers as compared to normal prostate tissues [10]. Additional expression data from the same database revealed PNUTS mRNA upregulation also in other tumor-normal pairs, including skin, kidney, and brain tissues. An immunohistochemical study also demonstrated PNUTS upregulation in esophageal carcinomas 


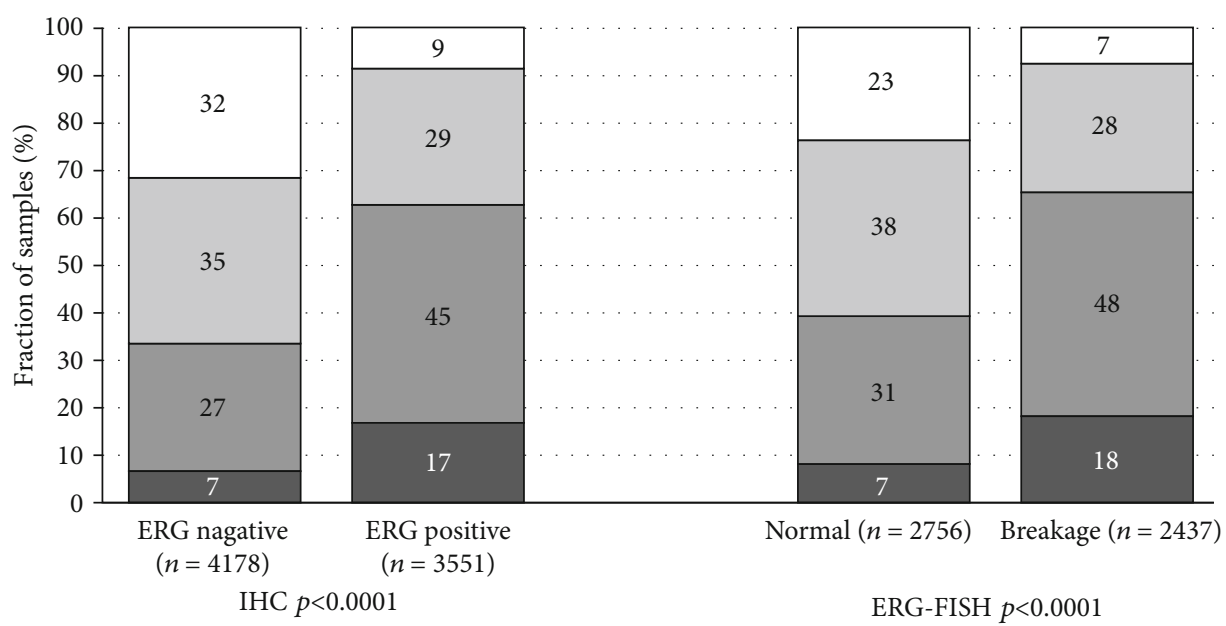

PNUTS

$\begin{array}{ll}\square \text { Negative } & \square \text { Moderate } \\ \square \text { Weak } & \square \text { Strong }\end{array}$

FIgURE 3: PNUTS staining and ERG status (IHC/FISH).

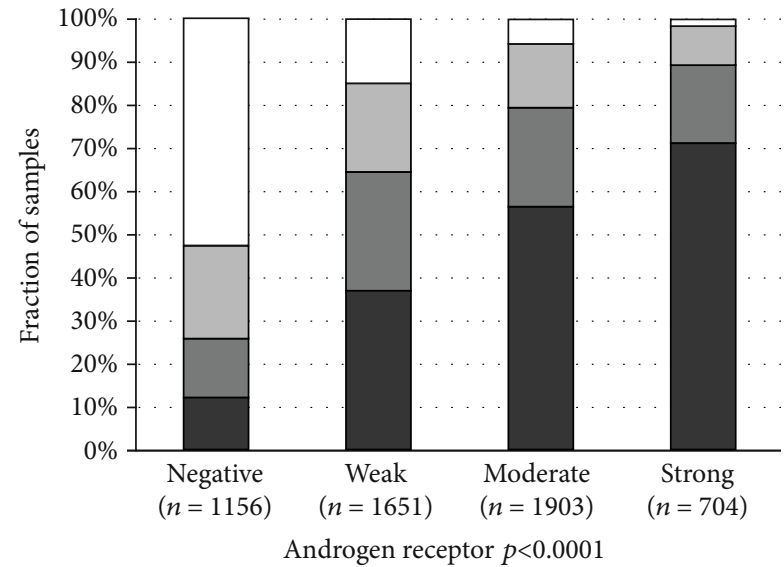

PNUTS

$\begin{array}{ll}\square \text { Negative } & \square \text { Moderate } \\ \square \text { Weak } & \square \text { Strong }\end{array}$

FIGURE 4: PNUTS staining and androgen receptor expression.

[10]. Altogether, these data suggest that PNUTS upregulation may accompany neoplastic transformation in many tumor types.

The association of high PNUTS staining with adverse tumor features, including advanced stage, high Gleason grade, nodal metastases, and early biochemical recurrence, argues for a role of PNUTS overexpression in prostate cancer progression. PNUTS is a protein that has not been extensively studied in cancer. A Medline search on $9^{\text {th }}$ March 2019 using the terms PNUTS and CANCER resulted in 19 citations. Data on a potential prognostic role of PNUTS activity in other cancer types is thus largely lacking. Kavela et al. reported that immunohistochemical PNUTS expression was stronger in 20 cases of triple-positive breast cancers that in 20 triple-negative breast cancers [10]. Overall, the known
TABLE 3: PNUTS staining correlates with Ki67 labeling index.

\begin{tabular}{|c|c|c|c|c|}
\hline Gleason & PNUTS & $N$ & Ki67LI $($ mean \pm SEM $)$ & $p$ \\
\hline \multirow{4}{*}{ All } & Negative & 1,181 & $1.74 \pm 0.07$ & \multirow{4}{*}{$<0.0001$} \\
\hline & Weak & 1,695 & $2.71 \pm 0.06$ & \\
\hline & Moderate & 1,831 & $3.02 \pm 0.06$ & \\
\hline & Strong & 598 & $3.82 \pm 0.10$ & \\
\hline \multirow{4}{*}{$\leq 3+3$} & Negative & 386 & $1.46 \pm 0.10$ & \multirow{4}{*}{$<0.0001$} \\
\hline & Weak & 349 & $2.42 \pm 0.11$ & \\
\hline & Moderate & 345 & $2.43 \pm 0.11$ & \\
\hline & Strong & 92 & $3.41 \pm 0.21$ & \\
\hline \multirow{4}{*}{$3+4$} & Negative & 622 & $1.69 \pm 0.09$ & \multirow{4}{*}{$<0.0001$} \\
\hline & Weak & 968 & $2.54 \pm 0.07$ & \\
\hline & Moderate & 1,065 & $2.86 \pm 0.07$ & \\
\hline & Strong & 336 & $3.55 \pm 0.12$ & \\
\hline \multirow{4}{*}{$3+4$ tertiary 5} & Negative & 32 & $1.75 \pm 0.42$ & \multirow{4}{*}{0.0019} \\
\hline & Weak & 82 & $3.35 \pm 0.26$ & \\
\hline & Moderate & 70 & $3.71 \pm 0.29$ & \\
\hline & Strong & 14 & $2.93 \pm 0.64$ & \\
\hline \multirow{4}{*}{$4+3$} & Negative & 78 & $2.29 \pm 0.36$ & \multirow{4}{*}{0.0009} \\
\hline & Weak & 150 & $3.41 \pm 0.26$ & \\
\hline & Moderate & 183 & $3.36 \pm 0.24$ & \\
\hline & Strong & 80 & $4.38 \pm 0.36$ & \\
\hline \multirow{4}{*}{$4+3$ tertiary 5} & Negative & 30 & $3.37 \pm 0.70$ & \multirow{4}{*}{0.1913} \\
\hline & Weak & 90 & $3.34 \pm 0.40$ & \\
\hline & Moderate & 105 & $4.35 \pm 0.37$ & \\
\hline & Strong & 42 & $4.45 \pm 0.59$ & \\
\hline \multirow{4}{*}{$\geq 4+4$} & Negative & 33 & $3.24 \pm 0.80$ & \multirow{4}{*}{0.0528} \\
\hline & Weak & 56 & $3.66 \pm 0.62$ & \\
\hline & Moderate & 61 & $4.97 \pm 0.59$ & \\
\hline & Strong & 33 & $5.88 \pm 0.80$ & \\
\hline
\end{tabular}


TABLE 4: Cox proportional hazards for PSA recurrence-free survival after prostatectomy of established preoperative prognostic parameter and PNUTS expression.

\begin{tabular}{lcccc}
\hline Variable & & $N$ & Univariable analysis & Multivariable analysis $(N=4,392)$ \\
\hline Gleason grade biopsy & $\geq 4+4$ vs. $\leq 3+3$ & 12,172 & $5.91(5.33-6.55)^{* * *}$ & $3.75(3.13-4.49)^{* * *}$ \\
cT stage & T2c vs. T1c & 14,404 & $2.15(1.72-2.65)^{* * *}$ & $2.00(1.46-2.74)^{* * *}$ \\
Preoperative PSA level & $\geq 20$ vs. $<4$ & 14,611 & $5.06(4.41-5.81)^{* * *}$ & $3.69(2.80-4.86)^{* * *}$ \\
PTEN & Deletion vs. normal & 6,236 & $2.10(1.89-2.33)^{* * *}$ & $1.44(1.26-1.65)^{* * *}$ \\
PNUTS expression & Strong vs. negative & 10,029 & $2.21(1.93-2.53)^{* * *}$ & $1.61(1.30-2.01)^{* * *}$ \\
\multicolumn{1}{c}{ ERG-negative subset } & Strong vs. negative & 4,342 & $2.70(2.17-3.36)^{* * *}$ & - \\
ERG-positive subset & Strong vs. negative & 3,811 & $2.16(1.67-2.83)^{* * *}$ & - \\
\hline
\end{tabular}

Confidence interval (95\%) in brackets; asterisks indicate significance level: ${ }^{*} p \leq 0.05,{ }^{* *} p \leq 0.001$, and ${ }^{* * *} p \leq 0.0001$.

interaction with key genes in cancer including PTEN and PP1 regulating also RB1, MYC, MDM2, p53, and Aurora kinases is consistent with an important role of PNUTS in cancer $[13,37-40]$.

Further of note, PNUTS maps to chromosome 6p21.3 within the major histocompatibility complex (MHC) class I gene cluster and contains binding intervals for the MHC master regulator CIITA [41]. It cannot be excluded that PNUTS overexpression may also impact antigen presentation and lead to altered immune responses against cancer.

The highly annotated molecular database enabled us to compare PNUTS expression with other relevant molecular alterations. About $50 \%$ of prostate cancers contain gene fusions connecting the androgen-regulated TMPRSS2 gene with the transcription factor $E R G[31,42]$. These fusions result in a high-level androgen-dependent expression of ERG [43] eventually leading to an altered expression of more than 1,600 genes in prostate epithelial cells [44]. The significant upregulation of PNUTS in cancers having a TMPRSS2:ERG fusion demonstrates that PNUTS is either directly or indirectly impacted by ERG expression. Possible explanations for this relationship include the known interaction of PNUTS with PTEN [10]. ERG expression suppresses the transcription of PTEN [45]. The significant association between androgen receptor and PNUTS expression fits well with earlier reports demonstrating interactions between the PNUTS target PP1 and the androgen receptor.

Next to TMPRSS2:ERG fusions, genomic deletions at various chromosomal loci represent the most prevalent recurrent genetic alterations in prostate cancer where specific mutations of cancer genes are rare [46] [47] [31] [48]. Most chromosomal deletions either preferentially occur in ERGnegative $(5 q, 6 q, 13 q$, and $18 q)$ or in ERG-positive $(3 p, 8 p$ PTEN (10q23), 12q, 16, and 17p) cancers. To exclude false statistical associations due to the link of both PNUTS expression and deletions to the ERG status, this analysis was also done for ERG-positive and ERG-negative cancers. That elevated PNUTS expression which was significantly associated with the majority of the analyzed deletions in ERG-negative cancers highlights that elevated PNUTS levels are either a cause or a consequence of genomic instability in prostate cancer cells. Given the pivotal interaction of PNUTS with PP1, an impact of PNUTS on the level of genomic stability appears plausible. Overexpression of NIPP1, another interac- tion partner of PP1, was earlier shown to limit a cell's capacity to repair DNA double-strand breaks [49]. Upregulation of PNUTS was most striking in cancers with PTEN and 12p13 deletions. In the case of PTEN, this may fit to the known functional interaction of both proteins. PNUTS binds directly to the PTEN protein and inactivates it through controlling its phosphatase activity. Knockdown of PNUTS in cell lines from prostate and other cancers resulted in apoptosis and growth arrest in a PTEN-dependent manner [10]. It could be hypothesized that high-level PNUTS expression may result in a particularly strong growth advantage in cancers with already reduced PTEN activity due to heterozygous deletion. However, this notion is not supported by the conspicuous lack of prognostic impact of PNUTS expression in 967 PTEN-deleted cancers. The tight relationship of elevated PNUTS expression and $12 \mathrm{p} 13$ deletions may pinpoint towards another relevant functional interaction. For example, ING4 maps to $12 \mathrm{p} 13$ and it is a tumor suppressor protein that contains a PHD-finger, which is a common motif in proteins involved in chromatin remodeling $[50,51]$. Similarly, CHD4 is also in this target deleted region and it is also involved in chromatin remodeling $[52,53]$. The data of this study identify high PNUTS expression as a strong and potentially clinically applicable prognostic marker in prostate cancer. The independent prognostic role of PNUTS expression was even retained if prognostic parameters were included, such as $\mathrm{pT}$ and $\mathrm{pN}$ stage, that are only available after prostatectomy and which are not known at the moment when therapeutic decisions are taken. That PNUTS expression which showed a prognostic impact in Gleason $3+4(p<0.0001)$ and Gleason $4+3$ carcinomas $(p=0.0002)$ demonstrates that these morphologically defined subgroups include cancers with highly variable aggressiveness. We had earlier shown that subdividing Gleason $3+4$ and $4+3$ cancers according to their percentage of Gleason 4 pattern results in a much finer prediction of tumor progression [21]. The limited prognostic role of PNUTS expression in cancers with comparable quantitative Gleason grade underscores the potential prognostic power of an optimized morphological prostate cancer assessment. This also demonstrates how difficult it is for a prognostic molecular marker to compete with an optimized assessment of morphological prostate cancer features. It is of note, however, that there is not only a need for better predictors of PCA aggressiveness than the existing ones but also 
for more reproducible parameters. The Gleason grade, for example, suffers from an interobserver variability in the range of $40 \%[54,55]$. This issue is not fully solved by a quantitative Gleason grading approach. We thus expect that molecular analysis including multiple different features will in the future improve prognosis assessment.

\section{Conclusions}

In summary, the results of our study demonstrate that upregulation of PNUTS is tightly linked to aggressive tumor behavior and poor prognosis in prostate cancer. Measuring PNUTS expression either alone or in combination with other prognostic markers might have clinical utility in prostate cancer.

\section{Data Availability}

Answer: No. Comment: The data used to support the findings of this study are available from the corresponding author upon request.

\section{Conflicts of Interest}

The authors declare that they have no competing interests.

\section{Authors' Contributions}

Guido Sauter, Jan Meiners, Markus Graefen, Thorsten Schlomm, and Ronald Simon were responsible for the conception and design. Christina Möller-Koop was responsible for the development of methodology. Andrea M Luebke, Doris Höflmayer, Jakob R. Izbicki, Franziska Büscheck, David Dum, Eike-Christian Burandt, Stefan Steurer, Maria Christina Tsourlakis, Cosima Göbel, Patrick Lebok, and Simon Kind were responsible for the acquisition of data (provided animals, acquired and managed patients, provided facilities, etc.). Ronald Simon and Guido Sauter were responsible for the analysis and interpretation of data (e.g., statistical analysis, biostatistics, and computational analysis). Ronald Simon, Guido Sauter, Hartwig Huland, Hans Heinzer, and Alexander Haese were responsible for the writing, review, and/or revision of the manuscript. Claudia Hube-Magg, Sarah Minner, Till S. Clauditz, and Christoph Fraune were responsible for the administrative, technical, or material support (i.e., reporting or organizing data, tissue processing, and antibody development). Guido Sauter, Ronald Simon, and Andreas Marx were responsible for the study supervision. Andreas Marx and Andreas M Luebke contributed equally.

\section{Acknowledgments}

We thank Christina Koop, Julia Schumann, Sünje Seekamp, and Inge Brandt for excellent technical assistance.

\section{Supplementary Materials}

Supplementary Table S1: PNUTS staining and prostate cancer phenotype in ERG-negative cancers. Supplementary
Table S2: PNUTS staining and prostate cancer phenotype in ERG-positive cancers. Supplementary Table S3: multivariate Cox regression analysis including established prognostic parameters and the PNUTS expression in all prostate cancers, the ERG-negative and ERG-positive subsets. Supplementary Fig. S1: association between PNUTS immunostaining and common chromosomal deletions in (a) all cancer, (b) ERG-negative cancers, and (c) ERG-positive cancers. Supplementary Fig. S2: prognostic impact of PNUTS defined by the Gleason score. (a) Impact of PNUTS expression as compared to the classical Gleason score categories. Impact of expression as compared to the quantitative Gleason score categories defined by subsets of cancers with (b) $\leq 5 \%$ Gleason 4 patterns, (c) 6-10\% Gleason 4 patterns, (d) $11-20 \%$ Gleason 4 patterns, (e) 21-30\% Gleason 4 patterns, (f) 3149\% Gleason 4 patterns, (g) 50-60\% Gleason 4 patterns, and (h) $\geq 61 \%$ Gleason 4 patterns. (Supplementary Materials)

\section{References}

[1] F. Bray, J. Ferlay, I. Soerjomataram, R. L. Siegel, L. A. Torre, and A. Jemal, "Global cancer statistics 2018: GLOBOCAN estimates of incidence and mortality worldwide for 36 cancers in 185 countries," CA: a Cancer Journal for Clinicians, vol. 68, no. 6, pp. 394-424, 2018.

[2] M. J. Resnick, "Re: use of active surveillance or watchful waiting for low-risk prostate cancer and management trends across risk groups in the United States, 2010-2015," Journal of Urology, vol. 202, no. 3, pp. 451-452, 2019.

[3] S. Grelet, L. A. Link, B. Howley et al., "A regulated PNUTS mRNA to lncRNA splice switch mediates EMT and tumour progression," Nature Cell Biology, vol. 19, no. 9, pp. 1105$1115,2017$.

[4] P. B. Allen, Y. G. Kwon, A. C. Nairn, and P. Greengard, "Isolation and characterization of PNUTS, a putative protein phosphatase 1 nuclear targeting subunit," The Journal of Biological Chemistry, vol. 273, no. 7, pp. 4089-4095, 1998.

[5] S. Rebelo, M. Santos, F. Martins, E. F. da Cruz e Silva, and O. A. da Cruz e Silva, "Protein phosphatase 1 is a key player in nuclear events," Cellular Signalling, vol. 27, no. 12, pp. 25892598, 2015.

[6] Y. M. Kim, T. Watanabe, P. B. Allen et al., "PNUTS, a protein phosphatase 1 (PP1) nuclear targeting subunit. Characterization of its PP1- and RNA-binding domains and regulation by phosphorylation," The Journal of Biological Chemistry, vol. 278, no. 16, pp. 13819-13828, 2003.

[7] H. B. Landsverk, F. Mora-Bermúdez, O. J. B. Landsverk et al., "The protein phosphatase 1 regulator PNUTS is a new component of the DNA damage response," EMBO Reports, vol. 11, no. 11, pp. 868-875, 2010.

[8] H. B. Landsverk, M. Kirkhus, M. Bollen, T. Küntziger, and P. Collas, "PNUTS enhances in vitro chromosome decondensation in a PP1-dependent manner," The Biochemical Journal, vol. 390, Part 3, pp. 709-717, 2005.

[9] H. Kim, O. H. Lee, H. Xin et al., "TRF2 functions as a protein hub and regulates telomere maintenance by recognizing specific peptide motifs," Nature Structural \& Molecular Biology, vol. 16, no. 4, pp. 372-379, 2009.

[10] S. Kavela, S. R. Shinde, R. Ratheesh et al., "PNUTS functions as a proto-oncogene by sequestering PTEN," Cancer Research, vol. 73, no. 1, pp. 205-214, 2013. 
[11] G. De Leon, T. C. Sherry, and N. A. Krucher, "Reduced expression of PNUTS leads to activation of Rb-phosphatase and caspase-mediated apoptosis," Cancer Biology \& Therapy, vol. 7, no. 6, pp. 833-841, 2008.

[12] M. S. Choy, M. Hieke, G. S. Kumar et al., "Understanding the antagonism of retinoblastoma protein dephosphorylation by PNUTS provides insights into the PP1 regulatory code," Proceedings of the National Academy of Sciences of the United States of America, vol. 111, no. 11, pp. 4097-4102, 2014.

[13] D. Dingar, W. B. Tu, D. Resetca et al., "MYC dephosphorylation by the PP1/PNUTS phosphatase complex regulates chromatin binding and protein stability," Nature Communications, vol. 9, no. 1, p. 3502, 2018.

[14] F. Wang, L. Wang, L. A. Fisher, C. Li, W. Wang, and A. Peng, "Phosphatase 1 nuclear targeting subunit (PNUTS) regulates Aurora kinases and mitotic progression," Molecular Cancer Research, vol. 17, no. 1, pp. 10-19, 2019.

[15] P. G. Santamaria, G. Moreno-Bueno, F. Portillo, and A. Cano, "EMT: present and future in clinical oncology," Molecular Oncology, vol. 11, no. 7, pp. 718-738, 2017.

[16] G. De Leon, M. Cavino, M. D'Angelo, and N. A. Krucher, "PNUTS knockdown potentiates the apoptotic effect of Roscovitine in breast and colon cancer cells," International Journal of Oncology, vol. 36, no. 5, pp. 1269-1275, 2010.

[17] K. Jin, Y. Xiang, J. Tang et al., "miR-34 is associated with poor prognosis of patients with gallbladder cancer through regulating telomere length in tumor stem cells," Tumour Biology, vol. 35, no. 2, pp. 1503-1510, 2014.

[18] H. Huang, H. Tian, Z. Duan, Y. Cao, X. S. Zhang, and F. Sun, "MicroRNA-383 impairs phosphorylation of H2AX by targeting PNUTS and inducing cell cycle arrest in testicular embryonal carcinoma cells," Cellular Signalling, vol. 26, no. 5, pp. 903-911, 2014.

[19] F. Wang, S. Zhu, L. A. Fisher et al., "Phosphatase 1 nuclear targeting subunit mediates recruitment and function of poly (ADP-ribose) polymerase 1 in DNA repair," Cancer Research, vol. 79, no. 10, pp. 2526-2535, 2019.

[20] D. R. Rhodes, J. Yu, K. Shanker et al., "ONCOMINE: a cancer microarray database and integrated data-mining platform," Neoplasia, vol. 6, no. 1, pp. 1-6, 2004.

[21] G. Sauter, S. Steurer, T. S. Clauditz et al., "Clinical utility of quantitative Gleason grading in prostate biopsies and prostatectomy specimens," European Urology, vol. 69, no. 4, pp. 592-598, 2016.

[22] J. Kononen, L. Bubendorf, A. Kallioniemi et al., "Tissue microarrays for high-throughput molecular profiling of tumor specimens," Nature Medicine, vol. 4, no. 7, pp. 844-847, 1998.

[23] R. Simon, "The impact of the number of cores on tissue microarray studies investigating prostate cancer biomarkers," International Journal of Oncology, vol. 40, pp. 261-268, 2011.

[24] S. Minner, M. Enodien, H. Sirma et al., "ERG status is unrelated to PSA recurrence in radically operated prostate cancer in the absence of antihormonal therapy," Clinical Cancer Research, vol. 17, no. 18, pp. 5878-5888, 2011.

[25] A. Krohn, A. Seidel, L. Burkhardt et al., "Recurrent deletion of 3 p13 targets multiple tumour suppressor genes and defines a distinct subgroup of aggressive ERG fusion-positive prostate cancers," The Journal of Pathology, vol. 231, no. 1, pp. 130141, 2013.

[26] L. Burkhardt, S. Fuchs, A. Krohn et al., "CHD1 is a 5 q21 tumor suppressor required for ERG rearrangement in prostate cancer," Cancer Research, vol. 73, no. 9, pp. 2795-2805, 2013.
[27] M. Kluth, J. Hesse, A. Heinl et al., "Genomic deletion of MAP3K7 at 6q12-22 is associated with early PSA recurrence in prostate cancer and absence of TMPRSS2:ERG fusions," Modern Pathology, vol. 26, no. 7, pp. 975-983, 2013.

[28] M. Kluth, N. N. Amschler, R. Galal et al., "Deletion of $8 p$ is an independent prognostic parameter in prostate cancer," Oncotarget, vol. 8, no. 1, pp. 379-392, 2017.

[29] A. Krohn, T. Diedler, L. Burkhardt et al., "Genomic deletion of PTEN is associated with tumor progression and early PSA recurrence in ERG fusion-positive and fusion-negative prostate cancer," The American Journal of Pathology, vol. 181, no. 2, pp. 401-412, 2012.

[30] M. Kluth, R. Ahrary, C. Hube-Magg et al., "Genomic deletion of chromosome $12 \mathrm{p}$ is an independent prognostic marker in prostate cancer," Oncotarget, vol. 6, no. 29, pp. 27966-27979, 2015.

[31] J. Weischenfeldt, R. Simon, L. Feuerbach et al., "Integrative genomic analyses reveal an androgen-driven somatic alteration landscape in early-onset prostate cancer," Cancer Cell, vol. 23, no. 2, pp. 159-170, 2013.

[32] M. Kluth, S. Scherzai, F. Büschek et al., "13q deletion is linked to an adverse phenotype and poor prognosis in prostate cancer," Genes, Chromosomes \& Cancer, vol. 57, no. 10, pp. 504$512,2018$.

[33] M. Kluth, S. Jung, O. Habib et al., "Deletion lengthening at chromosomes $6 \mathrm{q}$ and $16 \mathrm{q}$ targets multiple tumor suppressor genes and is associated with an increasingly poor prognosis in prostate cancer," Oncotarget, vol. 8, no. 65, pp. 108923108935, 2017.

[34] M. Kluth, S. Harasimowicz, L. Burkhardt et al., "Clinical significance of different types of p53 gene alteration in surgically treated prostate cancer," International Journal of Cancer, vol. 135, no. 6, pp. 1369-1380, 2014.

[35] M. Kluth, M. Graunke, C. Möller-Koop et al., "Deletion of $18 \mathrm{q}$ is a strong and independent prognostic feature in prostate cancer," Oncotarget, vol. 7, no. 52, pp. 86339-86349, 2016.

[36] J. P. Kreivi, L. Trinkle-Mulcahy, C. E. Lyon, N. A. Morrice, P. Cohen, and A. I. Lamond, "Purification and characterisation of p99, a nuclear modulator of protein phosphatase 1 activity," FEBS Letters, vol. 420, no. 1, pp. 57-62, 1997.

[37] J. V. Egger, M. V. Lane, L. A. Antonucci, B. Dedi, and N. A. Krucher, "Dephosphorylation of the Retinoblastoma protein $(\mathrm{Rb})$ inhibits cancer cell EMT via Zeb," Cancer Biology \& Therapy, vol. 17, no. 11, pp. 1197-1205, 2016.

[38] S. J. Lee, C. J. Lim, J. K. Min et al., "Protein phosphatase 1 nuclear targeting subunit is a hypoxia inducible gene: its role in post-translational modification of p53 and MDM2," Cell Death and Differentiation, vol. 14, no. 6, pp. 11061116, 2007.

[39] Z. Lu, G. Wan, H. Guo, X. Zhang, and X. Lu, "Protein phosphatase 1 inhibits p53 signaling by dephosphorylating and stabilizing Mdmx," Cellular Signalling, vol. 25, no. 4, pp. 796-804, 2013.

[40] J. C. Meadows, "Interplay between mitotic kinesins and the Aurora kinase-PP1 (protein phosphatase 1) axis," Biochemical Society Transactions, vol. 41, no. 6, pp. 1761-1765, 2013.

[41] D. Wong, W. Lee, P. Humburg et al., "Genomic mapping of the MHC transactivator CIITA using an integrated ChIP-seq and genetical genomics approach," Genome Biology, vol. 15, no. 10, p. 494, 2014. 
[42] S. A. Tomlins, D. R. Rhodes, S. Perner et al., "Recurrent fusion of TMPRSS2 and ETS transcription factor genes in prostate cancer," Science, vol. 310, no. 5748, pp. 644-648, 2005.

[43] J. P. Clark and C. S. Cooper, "ETS gene fusions in prostate cancer," Nature Reviews. Urology, vol. 6, no. 8, pp. 429-439, 2009.

[44] J. C. Brase, M. Johannes, H. Mannsperger et al., "TMPRSS2ERG -specific transcriptional modulation is associated with prostate cancer biomarkers and TGF- $\beta$ signaling," BMC Cancer, vol. 11, no. 1, article 2994, 2011.

[45] P. Adamo, S. Porazinski, S. Rajatileka et al., "The oncogenic transcription factor ERG represses the transcription of the tumour suppressor gene PTEN in prostate cancer cells," Oncology Letters, vol. 14, no. 5, pp. 5605-5610, 2017.

[46] C. E. Barbieri, S. C. Baca, M. S. Lawrence et al., "Exome sequencing identifies recurrent SPOP, FOXA1 and MED12 mutations in prostate cancer," Nature Genetics, vol. 44, no. 6, pp. 685-689, 2012.

[47] C. Gerhauser, F. Favero, T. Risch et al., "Molecular evolution of early-onset prostate cancer identifies molecular risk markers and clinical trajectories," Cancer Cell, vol. 34, no. 6, pp. 9961011.e8, 2018

[48] M. F. Berger, M. S. Lawrence, F. Demichelis et al., "The genomic complexity of primary human prostate cancer," Nature, vol. 470, no. 7333, pp. 214-220, 2011.

[49] C. Winkler, R. Rouget, D. Wu, M. Beullens, A. van Eynde, and M. Bollen, "Overexpression of PP1-NIPP1 limits the capacity of cells to repair DNA double-strand breaks," Journal of Cell Science, vol. 131, no. 13, article jcs214932, 2018.

[50] J. C. Shen, M. Unoki, D. Ythier et al., "Inhibitor of growth 4 suppresses cell spreading and cell migration by interacting with a novel binding partner, liprin alpha1," Cancer Research, vol. 67, no. 6, pp. 2552-2558, 2007.

[51] A. Shatnawi, S. A. Malkaram, T. Fandy, and E. Tsouko, "Identification of the inhibitor of growth protein 4 (ING4) as a potential target in prostate cancer therapy," Molecular and Cellular Biochemistry, vol. 464, no. 1-2, pp. 153-167, 2020.

[52] R. Smith, H. Sellou, C. Chapuis, S. Huet, and G. Timinszky, "CHD3 and CHD4 recruitment and chromatin remodeling activity at DNA breaks is promoted by early poly(ADPribose)-dependent chromatin relaxation," Nucleic Acids Research, vol. 46, no. 12, pp. 6087-6098, 2018.

[53] D. H. Larsen, C. Poinsignon, T. Gudjonsson et al., "The chromatin-remodeling factor CHD4 coordinates signaling and repair after DNA damage," The Journal of Cell Biology, vol. 190, no. 5, pp. 731-740, 2010.

[54] R. V. Singh, S. R. Agashe, A. V. Gosavi, and K. R. Sulhyan, "Interobserver reproducibility of Gleason grading of prostatic adenocarcinoma among general pathologists," Indian Journal of Cancer, vol. 48, no. 4, pp. 488-495, 2011.

[55] K. A. Iczkowski and M. S. Lucia, "Current perspectives on Gleason grading of prostate cancer," Current Urology Reports, vol. 12, no. 3, pp. 216-222, 2011. 